\title{
BMJ Open Is acupuncture effective for knee osteoarthritis? A protocol for a systematic review and meta-analysis
}

\author{
Chuan-Yang Liu (D) , ${ }^{1,2}$ Jian-Feng Tu, ${ }^{3}$ Myeong Soo Lee, ${ }^{4}$ Ling-Yu Qi (D) , ${ }^{3}$ \\ Fang-Ting Yu, ${ }^{3}$ Shi-Yan Yan, ${ }^{3}$ Jin-Ling Li, ${ }^{3}$ Lu-Lu Lin, ${ }^{3}$ Xiao-Wan Hao, ${ }^{3}$ \\ Xin-Tong Su, ${ }^{3}$ Jing-Wen Yang (1), ${ }^{3}$ Li-Qiong Wang ${ }^{3}$
}

To cite: Liu C-Y, Tu J-F, Lee MS, et al. Is acupuncture effective for knee osteoarthritis? A protocol for a systematic review and meta-analysis. BMJ Open 2022;12:e052270. doi:10.1136/ bmjopen-2021-052270

- Prepublication history and additional supplemental material for this paper are available online. To view these files, please visit the journal online (http://dx.doi.org/10.1136/ bmjopen-2021-052270)

Received 13 April 2021 Accepted 14 December 2021

Check for updates

(C) Author(s) (or their employer(s)) 2022. Re-use permitted under CC BY-NC. No commercial re-use. See rights and permissions. Published by BMJ.

${ }^{1}$ School of Traditional Chinese Medicine, Beijing University of Chinese Medicine, Beijing, China ${ }^{2}$ School of Traditional Chinese Medicine, Capital Medical University, Beijing, China ${ }^{3}$ School of Acupuncture-

Moxibustion and Tuina, Beijing University of Chinese Medicine, Beijing, China

${ }^{4} \mathrm{KM}$ Science Research division, Korea Institute of Oriental Medicine, Daejon, South Korea

Correspondence to MD Li-Qiong Wang; wangliqiongwork@163.com and MD Jing-Wen Yang; yangjw0626@126.com

\section{ABSTRACT}

Introduction Knee osteoarthritis $(\mathrm{KOA})$ is one of the leading causes of disability. The effectiveness of acupuncture for treating KOA remains controversial. This protocol describes the method of a systematic review and meta-analysis evaluating the effectiveness and safety of acupuncture for treating KOA.

Methods and analysis Four English databases (PubMed, Embase, Cochrane Library databases and Web of Science) and four Chinese databases (China National Knowledge Infrastructure, Chinese Biomedical Literature Database, VIP Database for Chinese Technical Periodicals, and Wanfang) will be searched from the database inception to 1 September 2021. All randomised controlled trials related to acupuncture for KOA will be included. Extracted data will include publication details, basic information, demographic data, intervention details and patient outcomes. The primary outcome will be pain intensity. Risk of bias will be assessed using the Cochrane Collaboration's tool for assessing risk of bias. Article selection, data extraction and risk of bias assessment will be performed in duplicate by two independent reviewers. If the meta-analysis is precluded, we will conduct a descriptive synthesis using a best-evidence synthesis approach. The strength of recommendations and quality of evidence will be assessed using the Grading of Recommendations Assessment Development and Evaluation working group methodology. Ethics and dissemination Ethics approval is not required because individual patient data are not included. This protocol was registered in the international Prospective Register of Systematic Reviews on 25 February 2021. The systematic review and meta-analysis will be submitted for publication in a peer-reviewed journal. The findings will also be disseminated through conference presentations. Trial registration number CRD42021232177.

\section{INTRODUCTION}

\section{Description of the condition}

Osteoarthritis (OA) is a common clinical degenerative disease and is one of the leading causes of disability. ${ }^{1}$ The excess costs of adults with $\mathrm{OA}$ are considerable, estimated at $\$ 45$ billion annually in USA. ${ }^{2}$ Knee osteoarthritis (KOA) accounts for approximately $85 \%$ of global OA burden. ${ }^{3}$ With the trends of an ageing population and increasing obesity,

\section{Strengths and limitations of this study}

- This systematic review and meta-analysis will evaluate the effectiveness and safety of acupuncture in treating knee osteoarthritis by collecting comprehensive evidence.

- We want to focus on many different factors in subgroup analysis and to explore the applied law of different doses of acupuncture.

- This study will explore the difference in the effectiveness between manual acupuncture and electroacupuncture for knee osteoarthritis by synthesising the evidence from direct comparison and indirect comparison.

- We plan to search multiple Chinese and English language databases to ensure a comprehensive search of the literature.

- Transformation of pain scores will result in loss of some accuracy; however, we believe that it is clinically irrelevant.

the incidence of KOA is increasing for both sexes. ${ }^{45}$ In addition, pain symptoms associated with KOA result in physical and walking disability, which in turn give rise to an excess risk of all-cause mortality. ${ }^{67}$

Exercise and weight loss, two effective nonpharmacological treatments, are strongly recommended in all people with clinical OA. ${ }^{8}$ However, for patients with KOA, it is difficult to continue exercising and losing weight. Representatives of pharmacological interventions include analgesics and nonsteroidal anti-inflammatory drugs (NSAIDs). However, acetaminophen (paracetamol) is not associated with long-term pain improvement. ${ }^{9}$ Furthermore, many NSAIDs are associated with serious side effects such as cardiovascular, renal adverse effects and gastrointestinal bleeding. ${ }^{10}{ }^{11}$ In addition, the healthcare systems of Western countries are overstretched because of the increasing joint replacement requirements. ${ }^{12}$ In this context, identification of the efficacy of existing 
treatments or development of novel therapies remains an important priority.

\section{Description of the intervention}

Acupuncture has long been recognised as a nonpharmacological therapy in treating various disorders by inserting fine needles into specific anatomic points (acupoints) on the skin of the patient's body. As an important component of traditional Chinese medicine (TCM), acupuncture has been used in clinical practice for more than 3000 years.

The WHO has recommended acupuncture therapies for 107 diseases. The effectiveness of acupuncture for different kinds of pain diseases has been verified by a great deal of high-quality clinical trials. ${ }^{13-15}$ Recently, two individual patient data meta-analyses also reported that acupuncture was effective for the treatment of chronic pain, with treatment effects persisting over time. ${ }^{1617}$ In addition, acupuncture appears to be a safe intervention that has rare adverse effects in the hands of competent practitioners. $^{1819}$

\section{How the intervention might work}

KOA is a prevalent, chronic joint disorder, characterised by synovitis, overgrowth of subchondral bone, development of osteophytes, erosions and loss of the articular cartilage. Previous study found that cartilage damage is the origin and result of KOA. With the further study of KOA, synovitis has been verified to play a crucial part in the pathological development and the maintenance of pain in KOA. ${ }^{20}$

In recent decades, preclinical investigations of acupuncture mechanisms in KOA pain have increased. These studies show that acupuncture relieves symptoms of KOA by activating a variety of bioactive chemicals through peripheral, spinal and supraspinal mechanisms. ${ }^{21}$ For example, acupuncture can desensitise peripheral nociceptors and reduce proinflammatory cytokines peripherally and in the spinal cord. ${ }^{21-23}$ In addition, acupuncture dampens the transmission of noxious inputs at the spinal level with the involvement of spinal opioids, serotonin (ie, 5-hydroxytryptamine), norepinephrine, glial cell/ cytokines and signal molecules. ${ }^{2124-26}$ In addition, CBR1GABA-5-HT may be a novel pathway contributed to the effect of electroacupuncture (EA) on KOA pain. ${ }^{27} \mathrm{EA}$ downregulated IL-1 $\beta$ expression via activating the peripheral CBR2 to inhibit the KOA pain. ${ }^{28}$

\section{Why it is important to perform this review}

Research on acupuncture for KOA has been growing, but the findings have been inconsistent. Different guidelines do not reach an agreement on whether acupuncture should be recommended as an effective non-pharmacological treatment for KOA. ${ }^{89-31}$ In 2014, a clinical trial showed that acupuncture did not confer a benefit over sham treatment for pain or function. ${ }^{32}$ In 2019, however, a review suggested that acupuncture provided relief of pain associated with KOA. ${ }^{33}$
Most meta-analyses mainly focused on chronic pain and peripheral joint $\mathrm{OA}$ and were not specific to KOA. ${ }^{161734-37}$ Although there were some systematic reviews conducted to establish the association of acupuncture with KOA, few drew a definitive conclusion. ${ }^{38}{ }^{39}$ One systematic review has looked at the comparative effectiveness of manual acupuncture (MA) and EA, but considered only direct evidence. ${ }^{40}$ Furthermore, some rigorous randomised clinical trials (RCTs) in this field published within recent years were not included in previous systematic reviews. For example, a multicentre RCT published in 2020 by our team suggested that acupuncture had potential benefits for KOA. ${ }^{41}$ Thus, it is important to perform a systematic review and meta-analysis to inform clinical practice.

\section{Objectives}

We aim to evaluate the effectiveness and safety of acupuncture for treating patients with OA of the knee by conducting a systematic review and meta-analysis. For this purpose, we put forward the following questions about this review:

1. Is acupuncture effective for treating $\mathrm{OA}$ of the knee compared with sham control or no-acupuncture control?

2. Is there a difference in the effectiveness between MA and $\mathrm{EA}$ ?

\section{METHODS AND ANALYSIS}

\section{Patients and public involvement}

There will be no patients or public directly involved in this review. Only data already existent in the literature and the aforementioned sources will be used for this study.

\section{Protocol registration}

This protocol was registered in PROSPERO (CRD42021232177). It will be followed the standard methods of systematic review and meta-analysis. It will adher to the Preferred Reporting Items for Systematic Reviews and Meta-Analyses reporting guidelines (see online supplemental appendix 1). ${ }^{4243}$

\section{Criteria for including studies in this review \\ Types of studies}

RCTs (with or without blinding, including crossover design) of acupuncture therapy for KOA will be included. We will consider including older RCTs that were cited in previous reviews of acupuncture for OA.

\section{Types of participants}

Studies enrolling participants diagnosed as KOA will be included. The diagnostic criteria should be based on the American College of Rheumatology clinical criteria, National Institute for Health and Clinical Excellence guidelines or any other accepted guidelines. ${ }^{84}$ There will be no restrictions on their age, sex, race, education, economic status, Kellgren-Lawrence score or Outbridge score. $^{4546}$ 


\section{Types of interventions}

The eligible intervention is acupuncture including MA and EA. There will be no restriction on the sessions of acupuncture, needling techniques or stimulation methods.

\section{Types of control groups}

In this review, we plan to compare needle acupuncture with sham acupuncture, analgesic, usual care or waiting list control groups. Acupuncture plus one or more therapies with the same therapies also will be included.

\section{Outcomes}

Primary outcome

Pain intensity: The WOMAC Pain Subscale, Visual Analog Scale (VAS), Brief Pain Inventory, Numerical Rating Scale (NRS), Verbal Rating Scale or other validated outcome measures.

\section{Secondary outcomes}

1. Function: The WOMAC Function Subscale, Lysholm Scale or other validated scales.

2. Quality of life: The 12-Item Short Form Health Survey, 36-Item Short Form Health Survey, Assessment of Quality of Life Instrument or other validated scales.

3. Adverse events: incidence and severity of adverse events.

4. Drug use: number of people using emergency analgesics, frequency or dosage of medication for KOA.

5. Cost: incremental cost-effectiveness ratio of acupuncture treatment.

\section{Criteria for excluding studies in this review}

1. Participants with knee pain but no other criteria of KOA.

2. The intervention group received transcutaneous electrical nerve stimulation.

3. Studies reported only improvement rates.

4. Studies comparing one type of acupuncture with other type of acupuncture (except EA vs MA) and studies comparing acupuncture with complementary therapies or TCM.

\section{Search methods for identification of studies \\ Electronic searches}

We developed search strategies for four English databases (PubMed, Embase, Cochrane Library databases and Web of Science) and four Chinese databases (China National Knowledge Infrastructure, Chinese Biomedical Literature Database, VIP Database for Chinese Technical Periodicals, and Wanfang) from database inception to 1 September 2021. Additional trials will be identified by searching previous systematic reviews. No language or publication status restrictions are applied. The search strategy components are clinical condition (OA, chondromalacia patellae, knee, knee pain and gonarthrosis), intervention (acupuncture, EA and acupuncture points) and study type (RCT). We will adapt the search strategies to medical subject headings terms and keywords as necessary for each database (see online supplemental appendix 2 for the search strategy used in the PubMed database). A pilot of the systematic search was conducted on 28 February 2021 (see online supplemental appendix 3 ). We (F-TY and CL) will rerun the searches before submission of the manuscript to identify any eligible articles published since our first search.

\section{Searching other sources}

We will search the following websites as a supplement: the WHO International Clinical Trials Registry Platform and the National Institutes of Health clinical registry ClinicalTrials.gov and the Chinese Clinical Registry. The search will also include a manual search for grey literature (eg, unpublished conference articles).

\section{Data collection and analysis}

Selection of studies

All search results will be exported to EndNote, where we will check for and exclude duplicates. Two of us will screen all titles and abstracts independently to identify potentially relevant studies. Full texts will be downloaded and printed for further assessment. Two reviewers will screen the whole-length articles to confirm whether the studies meet the inclusion criteria. Any disagreement will be settled by discussion. If an agreement cannot be reached, a third reviewer will be consulted. The reasons for excluding studies will be recorded. The study selection process is shown in figure 1. Besides, we will add a table of excluded studies with reasons for exclusion to the appendix of our meta-analysis.

\section{Data extraction and management}

All data will be extracted independently and in duplicate by two reviewers with a predesigned data extraction template. Disagreements will be settled by discussion. A third reviewer will be consulted if discrepancies cannot be resolved. All data will be cross-checked by two reviewers and transferred into Microsoft Office Excel. If required, we will contact the corresponding authors for more information by email.

The predefined variables for extraction are the following:

1. Publication details (study year, first author, funding source).

2. Basic information (location, study type, number of centres, sample size, study duration and length of follow-up).

3. Participants (type and/or stage of KOA, mean age, sex and pain intensity before treatment).

4. Interventions (type of acupuncture, choice of acupuncture points, number of sessions, treatment frequency, duration of each session and needling techniques).

5. Control (if there is any control, details of the treatment, including the name, dosage, frequency and course).

6. Outcomes (data and time points for each measurement, type and number of adverse events in each group). 


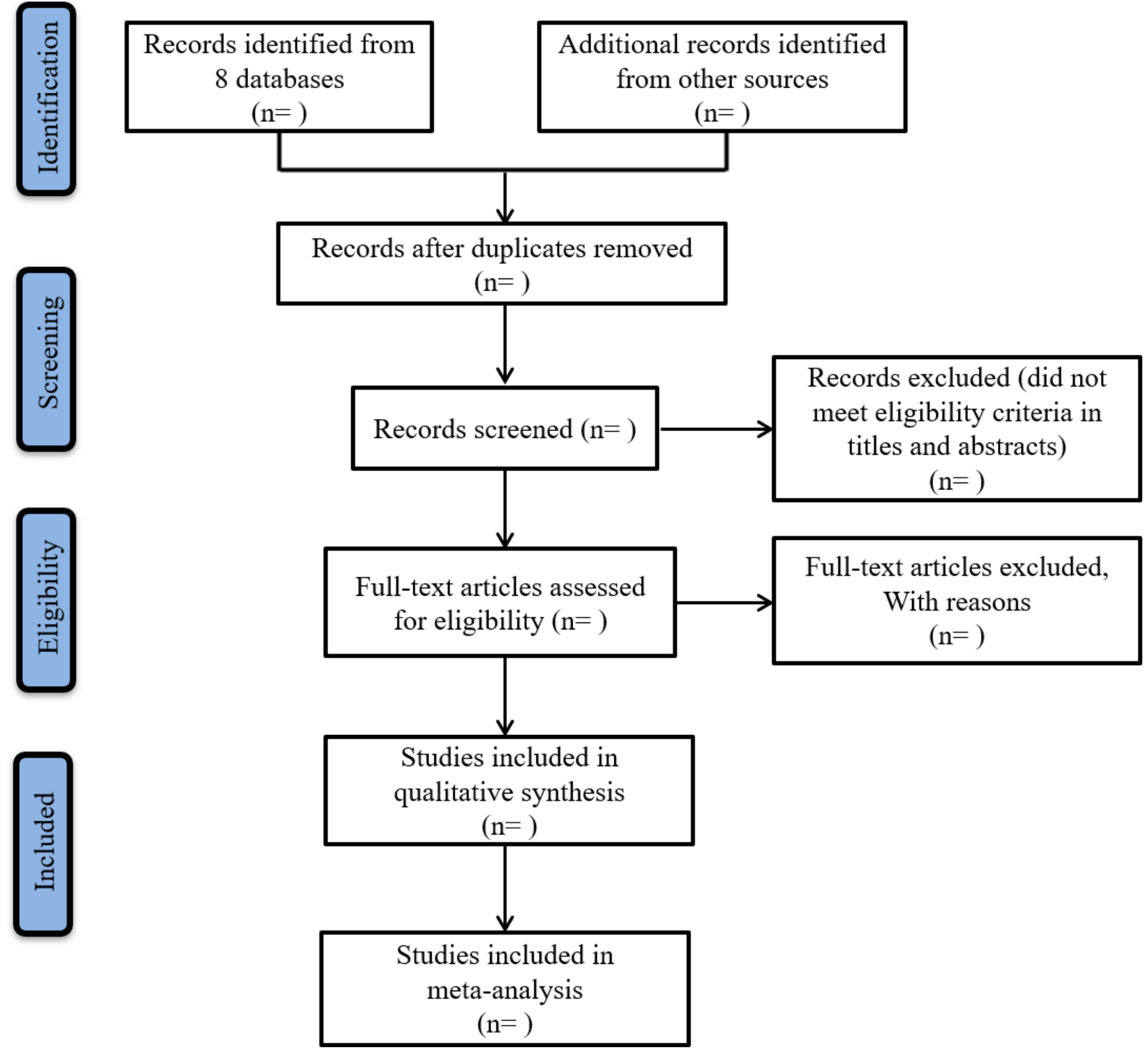

Figure 1 Flow diagram of the study selection process. KOA, knee osteoarthritis.

Risk-of-bias assessment in included studies

Two reviewers will assess the risk of bias in the included studies by using the Cochrane Collaboration's tool for assessing risk of bias. We will assess each RCT a low, high or unclear risk of bias for six domains: selection bias (random sequence generation and allocation concealment), performance bias (blinding of researchers and participants), attrition bias (incomplete outcome data), ascertainment bias (blinding of outcome assessment), reporting bias (selective outcome reporting) and other sources of potential bias. Disagreements will be resolved by discussion, according to the published articles and supplementary materials. We will consult the third reviewer and contact the study authors when needed.

\section{Acupuncture adequacy assessment}

We will use the adequacy assessment instrument to assess treatment adequacy in acupuncture RCTs from the following four aspects of acupuncture treatment: choice of acupuncture points, number of sessions, needling technique and experience of the acupuncturists. ${ }^{47}$ Two assessors who are experienced acupuncturists will assess adequacy independently and reach an agreement by discussion. They will be blinded to the results of the study and the publication and conduct the assessments only based on the description of the study population and the acupuncture procedure. To test the success of the blinding, we will ask the assessors to guess the identity of each study. 


\section{Heterogeneity assessment}

If there are sufficient data, we will conduct a meta-analysis to determine the effectiveness of acupuncture and the related factors. $\mathrm{I}^{2}$ testing will be used to quantify heterogeneity among the included studies. ${ }^{48}$ We will present summary estimates in forest plots. If the $\mathrm{I}^{2}$ is more than $50 \%$, we will explore the possible sources of heterogeneity via metaregression and subgroup analyses. If a metaanalysis is not appropriate, we will conduct a descriptive synthesis using a best-evidence synthesis approach.

\section{Reporting bias assessment}

We will also consider assessing the reporting bias and small-study effects by using funnel plots when there are 10 or more trials. We will assess funnel plot asymmetry by using Begg's and Egger's tests and will define significant publication bias as a $\mathrm{p}$ value $<0.1$. We will also use a trimand-fill computation to estimate the effect of publication bias on the interpretation of the results. ${ }^{49}$

\section{DATA SYNTHESIS}

When the meta-analysis is performed, Stata V.16.0 and RevMan V.5.3 will be used for all statistical calculations. All the analyses will be based on the random-effect model because the RCTs included by us may come from different populations. For dichotomous variables, the MantelHaenszel method will be used for analyses and effect size will be reported as relative risk with $95 \%$ CIs. For continuous variables, the inverse variance method will be used for analyses and treatment effect will be reported as mean difference with $95 \%$ CIs. The standardised MD with $95 \%$ CIs will be used if different scales are used to evaluate a predesigned outcome.

For pain variance, we plan to pool data from previous studies reporting VAS $100 \mathrm{~mm}$, VAS $10 \mathrm{~cm}$, and NRS by transforming it to a '0-100-pain measure' using an appropriate multiplier. We also intend to analyse pain intensity by independently reporting the aforementioned scales.

\section{SUBGROUP ANALYSIS}

Subgroup analyses will be performed to explain the heterogeneity. Predefined subgroups include the location of studies, the type of intervention, the dosage of acupuncture, the stage of KOA and the TCM types of KOA.

\section{SENSITIVITY ANALYSIS}

We will conduct a sensitivity analysis to verify the robustness of the review conclusions. We will consider removing one study at a time to observe its effect on heterogeneity and effect size. In addition, the meta-analysis will be repeated after studies with lack of allocation concealment are excluded.

\section{OTHER ANALYSIS}

If MA and eletroacupuncture are effective for $\mathrm{KOA}$ compared with sham acupuncture, we will conduct the exploratory research to compare the difference in the effectiveness between MA and EA by synthesising the evidence from direct comparison and indirect comparison. For direct comparison results, we will use Revman to analyse. For the indirect comparison, we will choose sham acupuncture as a common comparator and use $\mathrm{R}$ software to analyse. Finally, we will conduct a mixed treatment comparison meta-analysis to synthesise the evidence from direct comparison and indirect comparison.

\section{Strength of recommendations and the quality of evidence}

We will assess the strength of recommendations based on the Grading of Recommendations Assessment Development and Evaluation working group methodology. The two categories of weak/conditional evidence and strong evidence will be used.

We will also assess the quality of evidence. The quality of evidence will be assessed according to the domains of risk of bias, consistency, directness, precision and publication bias. The assessments will be adjudicated into four levels: high, moderate, low or very low. 5051

\section{DISCUSSION}

This systematic review will be performed based on previous studies of acupuncture for KOA. Conclusions drawn from this review may be beneficial to patients with KOA, clinicians and policy-makers. We will summarise and explain the characteristics and findings of the included studies by conducting a systematic narrative synthesis.

Based on the above, we want to conduct some exploratory studies. (1) Is there a difference in the effectiveness between MA and EA? (2) Is the effectiveness (if any) related to the stage of KOA according to the KellgrenLawrence score or Outbridge score, some characteristics of acupuncture (eg, treatment frequency), type of control group, measurement time points of outcomes or other variables?

MA and EA are the most commonly used acupuncture therapies. MA maintains a moderate dose of stimulation by lifting, inserting and twisting needles to acupoints. However, it is laborious and difficult to reach an agreement on standards because of the different needle techniques. EA, which is widely used in clinical practice, refers to the pulse current input to acupoints on the basis of needle acupuncture. This approach can accurately control the dose of stimulation and save labour. In clinical trials for pain conditions, better analgesia appears to be obtained when electrical stimulation is added to manual stimulation than with MA needle stimulation alone. ${ }^{52}$ However, the findings may not be generalisable because of the different pain types.

There are many factors affecting the effectiveness of acupuncture. One review presented 'the challenge of adequacy of dose' recently. ${ }^{33}$ Our group built a scoring 
instrument to calculate the dose of acupuncture from four parameters. ${ }^{53}$ Based on the sum of the scores, we defined three doses of acupuncture treatment: high dosage, medium dosage and low dosage. And we designed three subgroups according the three kinds of dosage to explore the relationship between doses of acupuncture and effectiveness. Deqi response is a comprehensive sensation of soreness, numbness, heaviness, aching at and around acupoints produced by manipulation of the needles. It plays a role in acupuncture dosage so it is only one dimension of our scoring instrument. On one hand, not all types of acupuncture need a Deqi response during sessions. For example, MA and EA are required to cause a Deqi response while wrist-ankle acupuncture is not. Furthermore, electrical stimulation can enhance Deqi response elicited by manipulation of needles. Based on the different duration of active stimulation, it is necessary to compare the effectiveness of MA and EA. ${ }^{52}$ On the other hand, Deqi response is more emphasised in China than Western countries. ${ }^{54}$

Acupuncture has both specific effects caused by intervention itself and non-specific effects including patientacupuncturist relationship, patient expectations, and so on. Sham acupuncture group has usually been set in order to eliminate non-specific effects. The sham acupuncture can be divided into superficial insertion and non-penetrating insertion at traditional acupuncture points or not. ${ }^{55}$ Superficial insertion is not a physiologically inert procedure and thus decreases the difference between groups. ${ }^{56}$ Therefore, more and more trials choose non-penetrating sham acupuncture at nonacupoints as control to minimise the physiological effects of sham acupuncture.

The proposed review has several strengths. We plan to search multiple Chinese and English language databases to ensure a comprehensive search of the literature. Any meta-analyses will be performed according to the Cochrane Handbook for Systematic Reviews of Interventions. A further strength is that stringent eligibility criteria will be applied to ensure the quality of the included RCTs. In addition, pain intensity was selected as the targeted outcome because it plays an important role in the pain management of KOA. Transformation of pain scores measured by different pain scales to a $0-100$ pain measure will result in loss of some accuracy; however, we believe that it is clinically irrelevant.

\section{Ethics and dissemination}

Ethics approval is not required because individual patient data are not included. This protocol was registered in the international Prospective Register of Systematic Reviews on 25 February 2021. The systematic review and meta-analysis will be submitted for publication in a peerreviewed journal. The findings will also be disseminated through conference presentations.

Acknowledgements The authors are grateful to Dr. Hong-ping Li and Dr. Mei Han for their helpful assistance.
Contributors $\mathrm{C}-\mathrm{YL}$ is the guarantor and first author of the protocol. C-YL and L-QW designed the systematic review. C-YL and L-LL drafted the manuscript. J-WY, J-FT, $\mathrm{L}-\mathrm{QW}$ and $\mathrm{M}-\mathrm{SL}$ provided help to design and edited the manuscript. C-YL and F-TY will independently screen the eligible studies. C-YL and X-WH will extract data from included articles. J-LL and J-FT will assess the risk of bias. C-YL and X-TS will assess acupuncture adequacy, strength of recommendations and the quality of evidence. C-YL, L-YQ and S-YY will finish data synthesis. L-QW will arbitrate any disagreements during the review. All authors have read the manuscript and approved the final publication of the protocol.

Funding This study was supported in part by the National Natural Science Foundation of China (grant number 82004223).

Competing interests None declared.

Patient consent for publication Not applicable.

Provenance and peer review Not commissioned; externally peer reviewed.

Supplemental material This content has been supplied by the author(s). It has not been vetted by BMJ Publishing Group Limited (BMJ) and may not have been peer-reviewed. Any opinions or recommendations discussed are solely those of the author(s) and are not endorsed by BMJ. BMJ disclaims all liability and responsibility arising from any reliance placed on the content. Where the content includes any translated material, BMJ does not warrant the accuracy and reliability of the translations (including but not limited to local regulations, clinical guidelines, terminology, drug names and drug dosages), and is not responsible for any error and/or omissions arising from translation and adaptation or otherwise.

Open access This is an open access article distributed in accordance with the Creative Commons Attribution Non Commercial (CC BY-NC 4.0) license, which permits others to distribute, remix, adapt, build upon this work non-commercially, and license their derivative works on different terms, provided the original work is properly cited, appropriate credit is given, any changes made indicated, and the use is non-commercial. See: http://creativecommons.org/licenses/by-nc/4.0/.

\section{ORCID iDs}

Chuan-Yang Liu http://orcid.org/0000-0001-6125-8601

Ling-Yu Qi http://orcid.org/0000-0002-7167-776X

Jing-Wen Yang http://orcid.org/0000-0002-7031-3446

\section{REFERENCES}

1 Hunter DJ, Bierma-Zeinstra S. Osteoarthritis. Lancet 2019;393:1745-59.

2 Zhao X, Shah D, Gandhi K, et al. Clinical, humanistic, and economic burden of osteoarthritis among noninstitutionalized adults in the United States. Osteoarthritis Cartilage 2019;27:1618-26.

3 GBD 2015 Disease and Injury Incidence and Prevalence Collaborators. Global, regional, and national incidence, prevalence, and years lived with disability for 310 diseases and injuries, 19902015: a systematic analysis for the global burden of disease study 2015. Lancet 2016;388:1545-602.

4 Prieto-Alhambra D, Judge A, Javaid MK, et al. Incidence and risk factors for clinically diagnosed knee, hip and hand osteoarthritis: influences of age, gender and osteoarthritis affecting other joints. Ann Rheum Dis 2014;73:1659-64.

5 Hunter DJ, March L, Chew M. Osteoarthritis in 2020 and beyond: a Lancet Commission. Lancet 2020;396:1711-2.

6 Nüesch E, Dieppe P, Reichenbach S, et al. All cause and disease specific mortality in patients with knee or hip osteoarthritis: population based cohort study. BMJ 2011;342:d1165.

7 Liu Q, Niu J, Huang J, et al. Knee osteoarthritis and all-cause mortality: the Wuchuan osteoarthritis study. Osteoarthritis Cartilage 2015;23:1154-7.

8 Osteoarthritis: care and management [clinical guideline CG177]. National Institute for Health and Clinical Excellence. Available: http:// www.nice.org.uk/guidance/CG1772019 [Accessed December 2020].

9 Gregori D, Giacovelli G, Minto C, et al. Association of pharmacological treatments with long-term pain control in patients with knee osteoarthritis: a systematic review and meta-analysis. JAMA 2018;320:2564-79.

10 Grosser T, Ricciotti E, FitzGerald GA. The cardiovascular pharmacology of nonsteroidal anti-inflammatory drugs. Trends Pharmacol Sci 2017;38:733-48.

11 Lanas Á, Carrera-Lasfuentes P, Arguedas Y, et al. Risk of upper and lower gastrointestinal bleeding in patients taking nonsteroidal anti-inflammatory drugs, antiplatelet agents, or anticoagulants. Clin Gastroenterol Hepatol 2015;13:906-12. 
12 Hunter DJ. Osteoarthritis management: time to change the Deck. $J$ Orthop Sports Phys Ther 2017;47:370-2.

13 Hershman DL, Unger JM, Greenlee H, et al. Effect of acupuncture vs sham acupuncture or Waitlist control on joint pain related to aromatase inhibitors among women with early-stage breast cancer: a randomized clinical trial. JAMA 2018;320:167-76.

14 Xu S, Yu L, Luo X, et al. Manual acupuncture versus sham acupuncture and usual care for prophylaxis of episodic migraine without aura: multicentre, randomised clinical trial. $B M J$ 2020;368:m697.

15 Zhao L, Li D, Zheng $\mathrm{H}$, et al. Acupuncture as adjunctive therapy for chronic stable angina: a randomized clinical trial. JAMA Intern Med 2019;179:1388-97.

16 Vickers AJ, Vertosick EA, Lewith G, et al. Acupuncture for chronic pain: update of an individual patient data meta-analysis. J Pain 2018;19:455-74.

17 MacPherson H, Vertosick EA, Foster NE, et al. The persistence of the effects of acupuncture after a course of treatment: a meta-analysis of patients with chronic pain. Pain 2017;158:784-93.

18 Furuse N, Shinbara $\mathrm{H}$, Uehara A, et al. A multicenter prospective survey of adverse events associated with acupuncture and moxibustion in Japan. Med Acupunct 2017;29:155-62.

19 Zhang J, Shang H, Gao X, et al. Acupuncture-related adverse events: a systematic review of the Chinese literature. Bull World Health Organ 2010;88:915-21.

20 Berenbaum F. Osteoarthritis as an inflammatory disease (osteoarthritis is not osteoarthrosis!). Osteoarthritis Cartilage 2013;21:16-21.

21 Zhang R, Lao L, Ren K, et al. Mechanisms of acupunctureelectroacupuncture on persistent pain. Anesthesiology 2014;120:482-503.

22 Chen H, Shao X, Li L, et al. Electroacupuncture serum inhibits TNF- $\alpha$-mediated chondrocyte inflammation via the Ras-Raf-MEK1/2-ERK1/2 signaling pathway. Mol Med Rep 2017:16:5807-14.

23 Zhang M, Guo H, Ma Y, et al. Acupoint sensitization is associated withincreased excitability and hyperpolarization-activated current (I) in C- but not A $\delta$-Type neurons. Neuroscience 2019;404:499-509.

24 Ahsin S, Saleem S, Bhatti AM, et al. Clinical and endocrinological changes after electro-acupuncture treatment in patients with osteoarthritis of the knee. Pain 2009;147:60-6.

25 Seo B-K, Sung W-S, Park Y-C, et al. The electroacupunctureinduced analgesic effect mediated by $5-\mathrm{HT} 1,5-\mathrm{HT} 3$ receptor and muscarinic cholinergic receptors in rat model of collagenase-induced osteoarthritis. BMC Complement Altern Med 2016;16:212.

26 Seo BK, Park DS, Baek YH. The analgesic effect of electroacupuncture on inflammatory pain in the rat model of collagenase-induced arthritis: mediation by opioidergic receptors. Rheumatol Int 2013;33:1177-83.

27 Yuan X-C, Zhu B, Jing X-H, et al. Electroacupuncture potentiates cannabinoid receptor-mediated descending inhibitory control in a mouse model of knee osteoarthritis. Front Mol Neurosci 2018;11:112.

28 Yuan X-C, Wang Q, Su W, et al. Electroacupuncture potentiates peripheral CB2 receptor-inhibited chronic pain in a mouse model of knee osteoarthritis. J Pain Res 2018;11:2797-808.

29 Clinical guidelines for osteoarthritis (2018 Edition). Chinese Journal of Orthopaedics 2018;38:705-15.

30 Kolasinski SL, Neogi T, Hochberg MC, et al. 2019 American College of Rheumatology/Arthritis Foundation guideline for the management of osteoarthritis of the hand, hip, and knee. Arthritis Rheumatol 2020;72:220-33.

31 Bannuru RR, Osani MC, Vaysbrot EE, et al. OARSI guidelines for the non-surgical management of knee, hip, and polyarticular osteoarthritis. Osteoarthritis Cartilage 2019;27:1578-89.

32 Hinman RS, McCrory P, Pirotta M, et al. Acupuncture for chronic knee pain: a randomized clinical trial. JAMA 2014;312:1313-22.

33 Paley CA, Johnson MI. Acupuncture for the relief of chronic pain: a synthesis of systematic reviews. Medicina 2019;56:6.
34 Li C, Pei Q, Chen Y, et al. The response-time relationship and covariate effects of acupuncture for chronic pain: a systematic review and model-based longitudinal meta-analysis. Eur $J$ Pain 2020;24:1653-65.

35 Manyanga T, Froese M, Zarychanski R, et al. Pain management with acupuncture in osteoarthritis: a systematic review and meta-analysis. BMC Complement Altern Med 2014;14:312.

36 Kwon YD, Pittler MH, Ernst E. Acupuncture for peripheral joint osteoarthritis: a systematic review and meta-analysis. Rheumatology 2006;45:1331-7.

37 Manheimer E, Cheng K, Linde K, et al. Acupuncture for peripheral joint osteoarthritis. Cochrane Database Syst Rev 2010:Cd001977.

38 Zhang Q, Yue J, Golianu B, et al. Updated systematic review and meta-analysis of acupuncture for chronic knee pain. Acupunct Med 2017:35:392-403.

39 Lin X, Huang K, Zhu G, et al. The effects of acupuncture on chronic knee pain due to osteoarthritis: a meta-analysis. J Bone Joint Surg Am 2016;98:1578-85.

40 Chen N, Wang J, Mucelli A, et al. Electro-acupuncture is beneficial for knee osteoarthritis: the evidence from meta-analysis of randomized controlled trials. Am J Chin Med 2017;45:965-85.

41 Tu J-F, Yang J-W, Shi G-X, et al. Efficacy of intensive acupuncture versus sham acupuncture in knee osteoarthritis: a randomized controlled trial. Arthritis Rheumatol 2021;73:448-58.

42 Moher D, Shamseer L, Clarke M, et al. Preferred reporting items for systematic review and meta-analysis protocols (PRISMA-P) 2015 statement. Syst Rev 2015;4:1.

43 Shamseer L, Moher D, Clarke M, et al. Preferred reporting items for systematic review and meta-analysis protocols (PRISMA-P) 2015: elaboration and explanation. BMJ 2015;349:g7647.

44 Hochberg MC, Altman RD, Brandt KD, et al. Guidelines for the medical management of osteoarthritis. Part II. osteoarthritis of the knee. American College of rheumatology. Arthritis Rheum 1995;38:1541-6.

45 Kellgren JH, Lawrence JS. Radiological assessment of osteoarthrosis. Ann Rheum Dis 1957;16:494-502.

46 Outerbridge RE. The etiology of chondromalacia patellae. J Bone Joint Surg Br 1961;43-B:752-7.

47 Manheimer E, Linde K, Lao L, et al. Meta-analysis: acupuncture for osteoarthritis of the knee. Ann Intern Med 2007;146:868-77.

48 Higgins JPT, Thompson SG. Quantifying heterogeneity in a metaanalysis. Stat Med 2002;21:1539-58.

49 Duval S, Tweedie R. Trim and fill: a simple funnel-plot-based method of testing and adjusting for publication bias in meta-analysis. Biometrics 2000;56:455-63.

50 Alonso-Coello P, Oxman AD, Moberg J, et al. GRADE evidence to decision (EtD) frameworks: a systematic and transparent approach to making well informed healthcare choices. 2: clinical practice guidelines. BMJ 2016;353:i2089.

51 Alonso-Coello P, Schünemann $\mathrm{HJ}$, Moberg J, et al. GRADE evidence to decision (EtD) frameworks: a systematic and transparent approach to making well informed healthcare choices. 1: introduction. BMJ 2016;353:i2016.

52 Langevin HM, Schnyer R, MacPherson H, et al. Manual and electrical needle stimulation in acupuncture research: pitfalls and challenges of heterogeneity. J Altern Complement Med 2015;21:113-28.

53 Sun N, Tu JF, Lin LL, et al. Correlation between acupuncture dose and effectiveness in the treatment of knee osteoarthritis: a systematic review. Acupunct Med 2019;37:261-7.

$54 \mathrm{Li} \mathrm{YM}$. Who has the final say on the dose of acupuncture? Comment on the article by Tu et al. Arthritis Rheumatol 2021;73:1089-90.

55 Ho RS, Wong CH, Wu JC, et al. Non-specific effects of acupuncture and sham acupuncture in clinical trials from the patient's perspective: a systematic review of qualitative evidence. Acupunct Med 2021;39:3-19.

56 Sun Y, Liu Y, Liu B, et al. Efficacy of acupuncture for chronic Prostatitis/Chronic pelvic pain syndrome: a randomized trial. Ann Intern Med 2021;174:1357-66. 